\title{
LE COMPLÉMENT HYPOTHÉTIQUE EN LATIN
}

Le complément du verbe à sens conditionnel n'est pas présenté, il n'est même pas mentionné dans la quasi-totalité des grammaires de la langue latine. Certains dictionnaires (en premier lieu Thesaurus linguae Latinae) et la Grammaire de R. Kühner-C. Stegmann-A. Thierfelder traitent de l'emploi des adverbes ita et aliter, ainsi que de la préposition in à sens conditionnel et également de l'usage de la formule ea condicione, tout en négligeant d'autres aspects du complément du verbe hypothétique.

Nous nous proposons, dans le présent article, de donner une vue d'ensemble du complément hypothétique (nommé, parfois, aussi «circonstant» hypothétique ${ }^{1}$ ), vue qui pourra être utile à l'approche théorique du complément hypothétique, en premier lieu des langues romanes, ensuite des autres idiomes indo-européens. Nous allons présenter le complément conditionnel en suivant son évolution historique.

En latin, le complément hypothétique apparaît autant dans les textes influencés par le latin populaire, que chez les auteurs cultivés. Il y en a deux catégories:

I. Le complément de manière-conditionnel (à sens assez général, sans marques particulières) et

II. Le complément hypothétique proprement dit.

I. Le complément de manière-conditionnel

Le complément de manière-conditionnel est d'un usage plus ancien que le complément hypothétique proprement dit. Il est exprimé par des adverbes, ou par des substantifs et des gérondifs à l'Ablatif, accompagnés ou non-accompagnés de la préposition in; le complément hypothétique est rarement rendu par des pronoms précédés de in. Souvent le complément est rendu par des substantifs ou des pronoms accompagnés de la préposition sine.

a) Les adverbes ont les sens: ainsi, alors, autrement (d'une autre manière), au contraire. Ces adverbes peuvent résumer le sens d'une proposition conditionnelle, ou temporelle-conditionnelle, placées d'habitude avant la proposition contenant l'adverbe qui adquiert le sens conditionnel. Parfois, la proposition renfermant l'adverbe de manière-conditionnel se trouve devant la subordonnée; ou devant la fausse principale à sens conditionnel, ou temporel-conditionnel.

Les adverbes latins qui peuvent recevoir le sens conditionnel sont: ita, tum, aliter, contra.

Ita, au sens de ainsi, rend, sous une forme concentrée, le sens d'une proposition conditionnelle. Ita est souvent employé, à cette valeur, aux époques préclassique, clas- 
sique et postclassique; à la basse époque, ce sont surtout les auteurs cultivés qui l'utilisent. En voici quelques exemples; dans les propositions associées on trouve:

1. le temps présent (de l'indicatif, du subjonctif-optatif), ou le futur simple, ou bien le futur antérieur (de l'indicatif ou de l'impératif):

- «postea scrobes facito...et facito de scrobe aqua in sulcum defluat: ita oleas serito!», Caton, Agr., 43, 1.

- «boues uti ualeant pabulum...amurca s pargito...: ita... morbus a berit.», Caton, Agr., 103.

- Voir également Cicéron, Diu., 2, 60 etc.

- Voici un passage de Tite-Live:

«auxilio uos, Campani, dignos censet senatus; sed ita uobiscum amicitiam institui par est, ne qua uetustior amicitia ac societas uioletur.», 7, 31, $2^{2}$.

On remarque, dans cette dernière citation, que ita est suivi d'une proposition en apposition («e qua amicitia...violetur»).

Pour d'autres occurrences de ita en contexte conditionnel, voir Columelle, 2, 10, 16; Gaudentius, Serm., 9, 38 etc. ${ }^{3}$

2. des temps du passé (indicatif, subjonctif-optatif), par exemple:

- «dominum iugulauit et ita discessit.», Bellum Hispaniense, 18, 4.

À voir a ussi Plaute, Stich., 209-10; Tite-Live, 22, 61, 5; G régoire de Tours, Glor. Mart., 5, p. 489, $31^{4}$.

Ainsi comme on le constate, dans les passages cités, ita résume le plus souvent les propositions exprimant une condition possible, ou une condition considérée comme réalisée.

Tum, au sens de alors, apparaît également dans un contexte conditionnel. Tum est, à l'origine, un adverbe à sens instrumental-de manière ${ }^{5}$; parfois il est employé en tant que corrélatif des propositions hypothétiques ${ }^{6}$; souvent il se substitue à une proposition conditionnelle.

Voici quelques exemples (dans l'ordre chronologique de l'apparition des textes):

- «Qui mihi, ubi ad uxores uentumst, tum fiunt senes!», Térence, Phorm., 1010.

- «Tibi si recta probanti placebis, tum non modo tete uiceris, sed omnes et omnia.», Cicéron, Tusc., 2, 63.

- «Si sciens fallo, tum me Iuppiter Optimus Maximus...pessimo leto afficias!», Tite-Live, 22, 53, $11^{7}$.

Aliter, au sens autrement, est lui aussi beaucoup utilisé en qualité de circonstant de manière-conditionnel. Les exemples peuvent être groupés selon les temps et les modes employés dans les propositions associées (voir le propos supra, dans le cas de l'adverbe ita).

Les occurrences renfermant l'adverbe aliter sont présentes tout le long de la latinité vivante. Aux époques postclassique et tardive, ce sont surtout les auteurs cultivés qui emploient l'adverbe aliter. 
Voici des passages de Plaute et de Cicéron:

- «Ten ego ueham?: tun hoc feras argentum aliter a me?», Plaute, Asin., 700.

- «epistulam ... nobis ... placuit, ut isti ante legerent; aliter enim fuissemus ... in hos inofficiosi.», Cicéron, Att., 13, $27,1$.

Voir, en outre, César, G., 5, 29, 2, passage où aliter équivaut à une proposition hypothétique construite au plus-que-parfait de l'optatif (pour condition irréelle), exactement comme dans le fragment supra (Cicéron, Att., 13, 27, 1).

Pour c e qui e st de l'emploi de l'adverbe a liter, voir a ussi Gaius, I nst., 3 , 46 ; Tertullien, Scorp., 4 etc. $^{8}$

Il est à souligner que aliter résume une proposition conditionnelle négative.

La proposition conditionnelle négative peut être également résumée par l'adverbe contra. Exempli gratia:

«quae (sc.: mens ) ... aut languescit ... aut contra tumescit.», Quintilien, Inst., 1, $2,18^{9}$.

Dans d'autres passages, contra prend la place de l'adverbe tum, exempli gratia:

«hostem esse... hominum morumque malorum, contra defensorem hominum morumque bonorum.», Lucilius, 1335.

Précisons a ussi que 1 es adverbes d ont n ous a vons parlé s ont c oncurrencés dans cette fonction (complément de manière-condition), à l'exception de contra, à partir de l'époque préclassique, par de syntagmes contenant des substantifs, ou des adverbes. C'est ainsi que l'adverbe ita est concurrencé par hoc modo, ou par isto modo; souvent, dans le latin populaire de la basse époque, SIC se substitue à l'adverbe ita. Tum entre en concurrence avec tunc; à l'époque tardive, dans le latin populaire, tum est remplacé tantôt par tunc, tantôt par les composés: $\boldsymbol{e c c u}$ '+tunc et intunc; aliter est, par étapes, remplacé par alio modo, diuerso modo et la formule populaire altero in modo. Voici un passage de Plaute:

«Hoc modo (sc.: existimo) ut molestus ne sis.», Truc., 919.

b) Le circonstant de manière-condition est également exprimé par des substantifs et des gérondifs, précédés ou non-précédés de la préposition in; rarement par des pronoms à l'Ablatif accompagnés de in.

Le sens de manière, assez général, du complément est évident dans des occurrences comme celle que nous venons de citer (Plaute, Truc., 919), ou comme dans le passage suivant de Cicéron:

«In eius modi re quisquam tam impudens reperietur qui ad alienam causam, inuitis iis quorum negotium est, accedere aut adspirare audeat?», Diu. in Caecil., 20.

Le syntagme in eius modi re (au sens de dans une situation/un procès de ce genre) peut équivaloir à l'expression quae cum ita sint, ou à sa variante: quod cum ita sit qui, dans un certain contexte, acquiert un sens conditionnel, dans un autre contexte - un sens causal, ou, toujours en fonction du contexte, un sens concessif ${ }^{10}$. 
Le sens assez général du complément est mis en évidence autant par l'emploi de l'Ablatif que par l'utilisation de la préposition in.

Le complément de manière-conditionnel a lterne parfois, $d$ ans $l e$ même passage, avec le complément de manière-concessif. Voici un passage de Laelius:

«Stantes plaudebant in re ficta: quid arbitramur in vera (sc.: re) facturos fuisse?», Cicéron, Lael., 24.

Dans cette citation, in re ficta c'est le complément à sens concessif; quant au complément in vera, celui-ci résume le sens d'une proposition hypothétique (condition irréelle).

Le sens du complément de manière-concessif, ou celui du complément de manièrecondition pouvait être précisé par des marques particulières, absentes pourtant dans le passage qu'on vient de citer (Lael., 24). Ces marques sont des adverbes tels que: etiam, quamvis ou tamen; ita et solum; des adjectifs comme nullus et solus ${ }^{11}$.

Le complément de manière-condition introduit par in est fréquent à toutes les époques, dans les deux registres importants (le registre populaire et le registre cultivé). En témoignent ces exemples:

- «in discordia... pax ciuilis esse non potest.», Cicéron, Ph., 7, 23;

à la traduction:

«La paix civile ne saurait exister dans les conditions de la discorde.»

- «Valeret hoc crimen in illa ueteri seueritate ac dignitate reipublicae.», Cicéron, Verr., 2, 5, 46.

Les compléments in illa ueteri severitate et (in illa ueteri) dignitate résument, dans cette dernière citation, une condition irréelle.

Voir également Pline, Nat., 13, $131^{12}$, Pétrone, 108, 1, St. Avit., p. 59, 23 etc.

Le complément au sens négatif (qui résume, en fait, une conditionnelle négative) est parfois accompagné de l'adverbe contra qui en souligne le sens négatif. Exempli gratia:

«... si modo unum omnes sentiant a c probent; contra in dissensione nullam se salutem perspicere.», César, G., 5, 31, 2.

Le sens conditionnel du complément est, parfois, précisé par l'emploi des adjectifs et des adverbes restrictifs (solus, modo, solum et, toujours plus fréquent dans le latin populaire des époques postclassique et tardive, 1 'adverbe tantum au sens de seulement). Voici un exemple de Varron:

"sermo non potest in uno homine solo esse, sed ubi oratio cum altero coniuncta.», L.L., 6, 64 .

Le gérondif à 1 'Ablatif e st é galement fréquent. L'Ablatif n on p répositionnel est attesté en général chez les auteurs cultivés. En voici des occurrences tirées de quelques historiens:

- «uigilando, agundo, bene consulendo prospere omnia cedunt.», Salluste, Cat., 52, 29.

- «Hic omnes spes opesque sunt, quas seruando patriam seruamus, dedendo ad necem patriam deserimus ac prodimus.», Tite-Live, 9, 4, 14. 
On remarque, dans ce dernier passage, l'existence des structures parallèles renfermant le gérondif à sens conditionnel; nous mentionnons aussi la présence du polyptote: seruando-seruamus.

L'Ablatif du gérondif précédé de in apparaît surtout dans le latin populaire, parfois chez les auteurs cultivés aussi. Voici un passage de Plaute:

«Multum in cogitando dolorem indipiscor.», Trin., 224.

Voir également Phèdre, 1, 15, 1; Pline, Nat., 12, 29 etc. ${ }^{13}$

Le complément rendu par le gérondif, précédé ou non-précédé de préposition, indique d'habitude une condition possible, ou une condition reconnue remplie. Rarement on exprime, à l'aide du gérondif, une condition irréelle. À voir ce passage du Pro Milone:

«Huius... interfector si esset, in confitendo ab iisne poenam timeret quos liberauisset?», 79 .

On rencontre également, pour exprimer la condition, une autre tournure: adjectif verbal en -nd- + substantif, le tout à l'Ablatif, précédé de in et aussi d'un adverbe restrictif. Voir un passage de Pétrone:

«puer ipse, quem uult, sequatur, ut sit illi saltem in eligendo fratre [salua] libertas.». 80, 5 .

c) Souvent le complément de manière-condition est rendu par des substantifs précédés de la préposition sine. Le prédicat de ces propositions est souvent négatif. Voici quelques exemples:

«Nion fit sine periclo facinus magnum nec memorabile.», Térence, H. T., 314.

Sine periclo équivaut, dans ce passage, à une conditionnelle négative (donc: si periculum non est).

À voir les exemples suivants de Cicéron:

- «Nemo vir magnus sine aliquo afflatu diuino umquam fuit.», Nat. D., 2, 167.

- «Ne illi quidem qui maleficio et scelere pascuntur possunt sine ulla particula iustitiae uiuere.», Off., 2, 40.

- Voir également Verrinae, 2, 5, 11 etc.

Voir aussi un passage de Pétrone:

«erimus sine tormentorum iniuria hilares.», 102, 13.

Dans cette dernière citation, sine tormentorum iniuria $=$ si tormentorum iniuria non est.

Parfois le complément se réfère à un être humain. À voir un passage de Laelius:

«Qui esset tantus fructus in prosperis rebus, nisi haberes qui illis aeque ac tu ipse gauderet? Aduersas uero ferre difficile esset, sine eo qui illas grauius etiam quam tu ferret.», Cicéron, Lael., 22.

Sine eo équivaut dans ces lignes à une conditionnelle: nisi esset is (amicus)... Rarement, sine introduit un gérondif: 
«nec sine canendo (sc.: tibia) tibicines ... dicti.», Varron, L. L., 6, $75^{14}$.

Parfois, le complément de manière-condition alterne dans les textes avec l'Ablatif Absolu de manière-condition. Exempli gratia :

«atque ita est a me consulatus peractus, ut nihil sine consilio senatus, nihil non approbante populo Romano egerim...», Cicéron, Pis., 7.

Le syntagme sine+l'Ablatif d'un nom peut être remplacé par des adjectifs niés comme inuitus, inscius, inuocatus. À voir le passage suivant de Satyricon:

«Nemo inuitus audit, cum cogitur aut cibum sumere, aut uiuere.», 111, 13.

II. Le complément hypothétique proprement dit.

Ce complément est de date plus récente que le complément de manière-conditionnel.

Le complément hypothétique proprement dit apparaît sous deux formes: a) les syntagmes anciens, d'Ablatif non-prépositionnel, comme hac condicione, ea condicione et hac lege (au sens de hac condicione) et b) les syntagmes renfermant des prépositions (il s'agit de sub, cum et in).

a) Les syntagmes anciens sont formés d'un nom (condicio ou lex) à l'Ablatif et de l'adjectif haec à l'Ablatif, alternant avec ista ou ea. Peu à peu on emploie l'Ablatif d'autres démonstratifs ou indéfinis, tels que eadem, quaedam. Plus tard apparaissent les adjectifs qualificatifs comme certa. Ces adjectifs acquièrent un sens restrictif, spécifique de l'idée de condition.

La fausse opposition qui existe entre le complément hypothétique et le prédicat de sa proposition est, parfois, mise en relief par l'emploi de certains adverbes adversatifs, tels que: sed, tamen = pourtant. (Voir le passage infra - Cicéron, Arch., 25; voir aussi Pétrone, 86, 5).

Ces syntagmes sont assez rarement utilisés au pluriel (his condicionibus; his legibus), pour exprimer le circonstant hypothétique ${ }^{15}$.

Le circonstant hypothétique de cette catégorie est d'habitude suivi de propositions subordonnées en apposition, introduites par si/si non, ut, ne, dum. Voici quelques exemples de l'époque préclassique:

- «Semper tibi promissum habeto hac lege, dum superes datis.», Plaute, Asin., 166. ${ }^{16}$

- «...Ea lege hoc adeo faciam, si facit//

Quod ego hunc (sc.: agere) aequom censeo.», Térence, H.T., 1054-55.

Pour le complément hypothétique ea lege, voir aussi Térence, Eun., 102; pour his legibus, voir Plaute, Aul., 155; ibid. 157 etc. ${ }^{17}$.

Pendant les siècles suivants, les expressions hac condicione, ea condicione et ea lege sont fréquentes, en particulier dans le latin cultivé. Exempli gratia:

- «eos uidebar ea accepisse condicione, $u t$ eos, quoad possem, incolumes patriae et parentibus conseruarem.», Rhetorica ad Herennium, 4, 34. 
- «...iubere ei praemium tribui, sed ea condicione, ne quid postea scriberet.», Cicéron, Arch., 25.

- À voir également Cicéron, De or., 1; 101 ; Salluste, Iug., 79, 8 etc.

Aux époques postclassique et tardive, l'expression hac condicione ou ea condicione remplace, par étapes, la formule hac legelea lege. En témoigne ce passage de Pétrone:

«omnes qui in testamento meo legata habent, praeter libertos meos, hac condicione percipient quae dedi, si corpus meum in partes conciderint et astante populo comederint,», 141, 2.

Ce dernier passage contient les mots d'Eumolpus, vieux poète vagabond ${ }^{18}$.

À voir aussi chez Pétrone l'emploi de la formule cum hac exceptione, si... non $(86,5)$ à la place de cum hac condicione.

Il est à préciser que le syntagme hac condicione, ea condicione commence à être utilisé, à partir même de l'époque de Cicéron, avec certaines prépositions: $s u b, c u m$, in (voir la discussion infra).

b) Les premières attestations du syntagme sub condicione semblent se trouver chez Tite-Live ${ }^{19}$. Cette expression est beaucoup employée aux époques postclassique et tardive.

L'absence des adjectifs, tels que: hac, ista, indique qu'il s'agit d'une locution propre au latin familier et populaire. Parfois on rencontre les adjectifs courants pour ces tournures: ea, quaedam ou des adjectifs qualificatifs, comme: certa, contraria.

La locution conditionnelle sub condicione est en général explicitée par des propositions en apposition (introduites par si/si non, $u t$, ne; l'apposition peut être également rendue par des tournures paratactiques).

Voici un passage de Tite-Live:

«... tantum licentiae nouem annis, quibus regnant, sumpsisse, ut nobis negent potestatem liberam suffragii... s e permissuros esse? "sub condicione", i nquit, " nos reficietis decimum tribunos" $\gg, 6,40,8$.

Voir aussi Pline, Ep., 8, 18, 4.

Le nouveau syntagme, plus précis que la formule sans préposition, est agréé par les juristes et employé par les ecclésiastiques aussi. Voici quelques exemples:

- «reo sub condicione obligato», Gaius, Dig., 46, 1, 70, 1;

- Voir également Gaius, Inst., 2, 200.

- «de seruanda pace... sub condicione... », Gregorius Magnus, Ep., 9, 4420.

Chez les auteurs influencés par le latin populaire, on découvre l'expression: $s u b$ condicionem. Par exemple:

- «sub contrariam condicionem obligationem mihi spopondisti.», I avolenus, D ig., $12,1,36$. 
- «...sub illa(m) condicionem, ut hoc... non bioletur sepulcrum.», Inscriptiones Christianae - Rossi, no. 1125 (584 p. Chr.) ${ }^{21}$.

La formule cum (ea) condicione est fréquente aux époques postclassique et tardive. Voir en premier lieu un passage de Sénèque l'Orateur:

«Antonium illi non uitam cum condicione promittere, sed mortem sub infamia quaerere.», Suas., 7, 11.

La précision de cette formule explique la haute fréquence de son emploi chez les juristes. En voici quelques occurrences chez Iulius Paulus:

- «...cum ea condicione ancillam emptam domino adquisitam, cum qua condicione uenisse proponeretur.», Dig., 40, 1, 23.

- «est admittendum, sed cum condicione ...», Dig., 16, 2, 9, 122 .

Rare est la formule in tali condicione - à voir le juriste Labeo, Dig., 28, 7, $20^{23}$.

Voir aussi Lex Visigotharum:

«si puella... ad... ingenuum u enerit in ea condicione, $u t$ eum sibi maritum adquirat.», $3,2,8^{24}$.

Une locution de date assez récente est cum eo, ut/quod. C'est une abréviation populaire de la formule cum ea condicione (ou: cum eo praeceptolpacto). La première attestation se trouve, vraisemblablement, dans la correspondance de Cicéron: «sit sane, quoniam ita tu uis, sed tamen cum eo, credo, quod sine peccato meo fiat.», Att., 6, 1, $7^{25}$.

La formule cum eo, ut est employée par Tite-Live, Columelle, Celsus. En témoignent les exemples suivants:

- «Lanuuinis ciuitas data sacraque sua reddita cum eo, ut aedes lucusque Sospitae Iunonis communis Lanuuinis municipibus cum populo Romano esset.», Tite-Live, $8,14,2$.

- Voir aussi Tite-Live, 36, 5, 3.

- «obsequar uoluntati tuae cum eo, ne dubites.», Columelle, $5,1,4$.

- Voir également Celsus, 6, 2 extr. $^{26}$.

Rarement est attestée la formule in hoc, si-voir Vulgata, Gen., 34, $15^{27}$.

Le complément conditionnel proprement dit indique d'habitude une condition possible.

\section{L'origine du complément hypothétique:}

Le complément hypothétique, autant le type de manière-conditionnel, que le complément conditionnel proprement dit, provient du complément instrumental-de manière. Cela résulte tout d'abord de l'analyse de la relation logique qui existe entre le complément et le prédicat de la proposition en discussion, ensuite de l'emploi de certains adverbes comme ita et contra, de l'emploi de l'Ablatif non-prépositionnel dans le vieux latin et de l'emploi de certaines prépositions (in, sine, sub, cum) dans les textes moins anciens. Le syntagme à sens conditionnel renferme parfois le substantif modus (voir Cicéron, Diu. in Caecil., 20 - exemple discuté supra). 


\section{La fonction syntaxique du complément hypothétique}

Le complément hypothétique est étroitement lié au prédicat de sa proposition, comme l'est d'ailleurs le complément de manière lui-même ${ }^{28}$. L'emploi de certaines prépositions: $c u m$, sub, sine, est, en fait, éloquent pour cette étroite dépendance du complément par rapport au prédicat.

Le complément de manière-conditionnel remplit la fonction d'attribut supplémentaire, exempli gratia:

«Multum in cogitando dolorem indipiscor.», Plaute, Trin., 224.

Le complément hypothétique proprement dit remplit également la fonction d'attribut supplémentaire, par exemple:

«...hac condicione percipient quae dedi, si...», Pétrone, 141, 2.

À voir aussi Cicéron, Arch., 25 etc.

En résumé, le complément hypothétique du verbe est assez fréquent dans les deux registres importants de la langue latine, à partir de l'époque préclassique.

Il y a deux catégories de complément hypothétique:

I. Le complément de manière-condition rendu par des adverbes, ou des expressions ayant le sens: ainsi (de cette manière), alors, autrement (d'une autre manière), au contraire.

Ce complément est également exprimé par des substantifs, ou des gérondifs, à l'Ablatif, accompagnés ou non-accompagnés de la préposition in et des adjectifs. Sine suivi de l'Ablatif rend aussi le complément de manière-condition. Les substantifs, les pronoms et les gérondifs peuvent être accompagnés de mots restrictifs comme solus ou solum, modo.

II. Le complément conditionnel proprement dit est de date plus récente que le complément de manière-condition.

On reconnaît facilement le complément de condition proprement dit à cause de l'emploi du substantif condicio, ou du substantif lex, accompagné d'un adjectif démonstratif. Peu à peu apparaît l'emploi de certains adjectifs indéfinis et des adjectifs qualificatifs. Tous ces adjectifs acquièrent un sens restrictif, spécifique de l'idée de condition.

L'évolution du latin populaire vers des syntagmes précédés de préposition, mais aussi la tendance générale à des expressions très précises, suivant le développement de différentes sciences (philosophie, grammmaire, éloquence, médecine, astronomie, théologie chrétienne etc.) et en premier lieu du droit romain, ont mené à l'usage prépositionnel de ces formules (le latin a eu recours, pour le complément hypothétique proprement dit, aux prépostions $s u b$, cum et, rarement, in).

Le complément de condition (tous les types) indique, d'habitude, une condition possible, ou une condition considérée comme réalisée.

Le complément de condition, autant le circonstant de manière-condition que celui conditionnel proprement dit, est un développement du complément de manière. 
Le complément de condition, étroitement lié au prédicat de sa proposition, remplit la fonction d'attribut supplémentaire.

Nous désirons également souligner la diversité des modalités pour rendre le complément hypothétique, diversité qu'on retrouve, par ailleurs, dans les langues romanes.

\section{Notes}

1 Voir M. Arrivé, F. Gadet, M. Galmiche, La grammaire d'aujourd'hui, Paris, 1986, p. 114: “On donne parfois le nom de 'circonstant' à tout élément exerçant dans la phrase une fonction circonstantielle, quelle qu'en soit la manifestation: adverbe simple ou composé (locution adverbiale), syntagme prépositionnel (ou, dans certains cas, non prépositionnel), proposition subordonnée."

2 Exemple tiré de R. Kühner-C. Stegmann-A. Thierfelder, Ausführliche Grammatik der lateinischen Sprache, Hannover, 1971, II-2, p. 250.

3 Exemples extraits de Thesaurus linguae Latinae, VII-2, fasc. 1, Leipzig, 1956, pag. 522, 1. 33 sqq.

4 Exemples extraits de Thesaurus linguae Latinae, VII-2, fasc. 1, op. cit., pp. 522-23.

5 Voir, sur cette question, R. Iordache, 'Cum'temporal, o 'cum'explicativo?, o Sobre la procedencia y los principales valores de la conjunción 'cum', dans "Helmantica", no. 92/93, Salamanque, 1979, pp. 272-3 et pp. 278-81.

6 Pour ce qui est de la haute fréquence des exemples de cet emploi de tum, voir R. Iordache, 'Cum'temporal, o 'cum'explicativo?, op. cit., p. 271.

7 Pour d'autres exemples voir Wilh. Freund-N. Theil, Grand dictionnaire de la langue latine, Paris, 1883 , vol. III, p. 498.

8 Exemples tirés du Thesaurus linguae Latinae, I, Leipzig, 1900, p. 1654, 1. 60 sqq.

9 Parfois contra résume une proposition concessive (voir Pétrone, 14, 5-6).

10 Pour ce qui est des sens du syntagme in eius modi re et de la proposition quae cum ita sint/quod cum ita sit, voir $\mathrm{R}$. Iordache, Le complément de concession chez Cicéron; la définition du complément de concession en latin, en “ iva antika”, vol. 49, Skopje, 1999, pp. 98-9.

11 Pour les marques qui indiquent la présence du complément de concession, voir R. Iordache, Le complément de concession chez Cicéron..., op. cit., pp. 74-76.

12 Exemples extraits du Thesaurus linguae Latinae, VII-1, fasc. 5, Leipzig, 1938, p. 781, 1. 56 sqq.

13 Exemples extraits du Thesaurus linguae Latinae, VII-1, fasc. 5, op. cit., p. 781, 1.81 sqq.

14 Exemple tiré de R. Kühner-C. Stegmann-A. Thierfelder, op. cit., II-1, p. 751 b.

15 Ces formules, à l'Ablatif pluriel, servent d'habitude à rendre le complément de manière, ou le complément d'accompagnement, ou bien le complément de conformité (exempli gratia: "iustis legibus et aequis condicionibus bellum componere", Velleius Paterculus, 2, 25, 1).

Exemple tiré de Thesaurus linguae Latinae, VII-2, fasc. 1, op. cit., p. 1242, 1. 76 sqq.

17 Exemple tiré de Thesaurus linguae Latinae, VII-2, fasc. 1, op. cit., p. 1242, 1. 76-80.

18 Pour ce qui est de la fréquence de la formule ea condicione aux époques postclassique et tardive, voir Thesaurus linguae Latinae, VI, Leipzig, 1909, p. 128, 1. 78 sqq.

19 Selon R. Kühner-C. Stegmann-A. Thierfelder, op. cit., II-1, p. 570, par. 108, point 3 b.

20 Exemples tirés de Thesaurus linguae Latinae, IV, op. cit., p. 129, 1. 1 sqq.

21 Exemples tirés de Thesaurus linguae Latinae, IV, op. cit., p. 129, 1. 29-30. Le dernier passage cité (des Inscriptions chretiennes) renferme toute sorte d'erreurs: illa $(m)$ au lieu de $e a(m) ; u t . . n o n$ au lieu de ne; bioletur à la place de violetur.

22 Les derniers exemples sont extraits de Thesaurus linguae Latinae, IV, op. cit., p. 129, 1. 31 sqq.

23 Exemple tire de Thesaurus linguae Latinae, VII-1, fasc. 5, op. cit., p. 781, 1. 71.

24. Exemple tiré de Thesaurus linguae Latinae, IV, op. cit., p. 129, 1.37 sqq.

25 Voir R. Kühner-C. Stegmann-A. Thierfelder, op. cit., II-2, p: 250. 
Pour ce qui est de la définition du complément de manière, voir $\mathrm{R}$. Iordache, Les subordonnées de manière en latin, Bref Plaidoyer pour la Syntaxe Historique, dans “ iva antika”, vol. 48, Skopje, 1998, pp. 52-54.

\section{Povzetek \\ DOPOLNILO POGOJNOSTI V LATINŠC̆INI}

Pogojno dopolnilo glagola je od preklasične dobe naprej precej pogosto $v$ dveh pomembnih zvrsteh latinščine. Obstajata dve skupini pogojnega dopolnila:

1. načinovno-pogojno dopolnilo, ki se izraža s prislovi ali izrazi s pomenom 'tako', 'takrat', 'drugače', 'nasprotno'. To dopolnilo se lahko izraža tudi s samostalnikom ali gerundijem v ablativu bodisi $\mathrm{s}$ predlogom in bodisi brez njega, prav tako sine $\mathrm{z}$ ablativom. Samostalnik, zaimek ali gerundij je lahko dodatno določen $z$ omejevalnimi besedami kot solus, solum ali modo.

2. Pravo pogojno dopolnilo je mlajše od načinovno-pogojnega. V njem nastopata samostalnika condicio ali lex s kazalnim zaimkom, sčasoma tudi z nedoločnim zaimkom ali pridevnikom. Vsi ti dobijo omejevalni pomen, značilen za pojem pogoja.

Razvoj ljudske latinščine v smer predložnih zvez, a tudi splošna težnja po natančnejšem izražanju, ki jo je povzročil razvoj različnih znanosti, zlasti rimskega prava, sta pripeljala do predložne rabe teh stalnih zvez (latinščina se je pri tem zatekala k predlogom $s u b$, cum, redko tudi in). Pogojno dopolnilo ( $v$ vseh tipih) navadno zaznamuje možen pogoj ali pogoj, ki ga imamo za uresničen. $\mathrm{V}$ obeh obravnavanih tipih se je razvil iz dopolnila načina. 